\title{
Posture recognition using an RGB-D camera : exploring 3D body modeling and deep learning approaches
}

\author{
Mohamed El Amine Elforaici*, Ismail Chaaraoui*, Wassim Bouachir*, Youssef Ouakrim* and Neila Mezghani* \\ ${ }^{*}$ Laboratoire de recherche en Imagerie et Orthopédie (LIO), TÉLUQ University, Montréal, Canada
}

\begin{abstract}
The emergence of RGB-D sensors offered new possibilities for addressing complex artificial vision problems efficiently. Human posture recognition is among these computer vision problems, with a wide range of applications such as ambient assisted living and intelligent health care systems. In this context, our paper presents novel methods and ideas to design automatic posture recognition systems using an RGB-D camera. More specifically, we introduce two supervised methods to learn and recognize human postures using the main types of visual data provided by an RGB-D camera. The first method is based on convolutional features extracted from 2D images. Convolutional Neural Networks (CNNs) are trained to recognize human postures using transfer learning on RGB and depth images. Secondly, we propose to model the posture using the body joint configuration in the $3 \mathrm{D}$ space. Posture recognition is then performed through SVM classification of 3D skeletonbased features. To evaluate the proposed methods, we created a challenging posture recognition dataset with a considerable variability regarding the acquisition conditions. The experimental results demonstrated comparable performances and high precision for both methods in recognizing human postures, with a slight superiority for the $\mathrm{CNN}$-based method when applied on depth images. Moreover, the two approaches demonstrated a high robustness to several perturbation factors, such as scale and orientation change.
\end{abstract}

Index Terms-RGB-D camera, depth imaging, posture recognition, SVM, CNN, transfer learning.

\section{INTRODUCTION}

Posture recognition is a central problem in computer vision, with a wide range of applications such as human-machine interaction, content-based video annotation, and especially intelligent video surveillance systems for ambient assisted living. During recent years, several research works tried to address the posture recognition problem following various machine vision methods and using different types of visual data. Traditional posture recognition systems used conventional cameras to build a body model from RGB images through background subtraction or geometric transformations [1]-[3]. These methods present inherent limitations and lack the flexibility to handle challenging cases, such as dynamic backgrounds, camera motion, and silhouette deformation. Moreover, analyzing bidimensional images to construct a 3D body model, such as in [3], is often a source of computational complexity.

The emergence of RGB-D cameras (e.g. Microsoft Kinect) allowed to exploit depth information in order to design less complex methods for modeling human postures. In this sense, recent works tried to extend $2 \mathrm{D}$ image features to the $3 \mathrm{D}$

\footnotetext{
*This research was supported by the Canada Research Chair on Biomedical Data Mining (950-231214)
}

domain for human body modeling. Most of these works relied on the Space Time Interest Points (STIPs) [4], an extension of the 2D interest points into the spatio-temporal domain.The STIP framework were implemented in different ways, especially using the Harris3D detector [4] to identify interest points and the HOGHOF descriptor [5] to describe the regions around the STIPs.

Instead of generalizing 2D image descriptors to the 3D space, a second line of works [6]-[8] propose to use the skeleton detector [13] to build high-level features characterizing the 3D configuration of the body. This representation was successfully applied for activity recognition as it conforms to the early Johansson's biological studies on how human understand actions [9].

This paper aims to explore several acquisition possibilities offered by an RGB-D camera to design novel methods and ideas for automatic posture recognition. Our work proceeds along the two directions described above to propose and analyze two methods for posture recognition. Firstly, we propose a posture recognition method from $2 \mathrm{D}$ images. Instead of using hand-crafted features such as STIPs, we train Convolutional Neural Networks (CNNs) on RGB and depth images to recognize body postures. To the best of our knowledge, this is the first study proposing to use CNNs for static pose recognition. We note that deep neural networks were previously used for hand pose and gesture recognition [10], [11]. Secondly, we propose a new method for body modeling using joint-based features as a high-level descriptor. The proposed method uses a single depth image to perform supervised classification based on the 3D skeleton spatial configuration. We finally provide an experimental evaluation of the proposed methods on a new posture RGB-D dataset created using the Microsoft Kinect v2 camera.

The rest of the paper is organized as follows: section II presents the proposed methods, section III provides experimental results and discussions. Finally, section IV concludes the paper.

\section{The Proposed Methods}

\section{A. Posture recognition using $2 D$ images and Convolutional Neural Network}

The proposed method is based on using 2D images representing human postures to adapt a pretrained CNN for our specific recognition problem. We use the AlexNet network [12], that has been pre-trained from the ImageNet database to classify images into 1000 object categories. Before using 
posture images to retrain the network, our dataset is first pre-processed by resizing the images according to the CNN input format and subtracting the background to extract the body silhouette. Silhouette segmentation is initially performed through body parsing on depth images [13]. The segmentation result is then mapped to RGB images, since the infrared and RGB sensors are geometrically calibrated.

Once the pre-processing step is completed, we use the resulting images to retrain the $\mathrm{CNN}$ for posture classification. Prior to the network training procedure, we fine-tune the model in order to adapt the network to the new dataset. Since, training is performed using a relatively small image collection, the weights on initial layers are frozen [14]. However, the last three layers were transferred to the new classification task by replacing them with a fully connected layer, a softmax layer, and a classification output layer [15]. The classification output layer was set according to the five defined posture classes: standing, bending, sitting, walking, and crouching. The main steps of the proposed method are summarized in figure 2 .

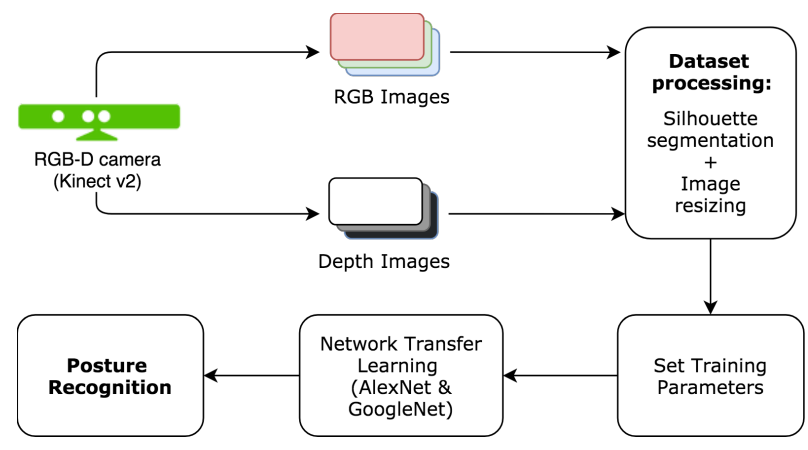

Fig. 1. Posture recognition using 2D images and CNN

\section{B. Posture recognition using $3 D$ joint-based features}

The second method is based on classifying high-level features representing the $3 \mathrm{D}$ body configuration. Firstly, a 3D skeleton model is built using the skeleton detector of Shotton et al. [13] for localizing different body parts and accurately model an articulated structure of connected segments. The obtained 3D skeleton formed by 25 joints represents the human posture as illustrated in the figure 2 . Once joint positions are extracted, we propose to compute 2 types of features representing human body posture: the 3D pairwise distances between joints, and the geometrical angles of adjacent segments.

Given the body skeleton obtained from depth images, we calculate the relative 3D distances between pairs of joints. Such features were successfully used to model human body for action recognition [6]. To ensure scale invariance, the $3 \mathrm{D}$ distances are normalized with respect to the person's height. The normalization process is performed by considering the 3D distance between the spine shoulder joint and the spine middle joint. This distance was chosen based on experimental observations concluding that it is sufficiently stable with respect to several body deformations, in addition to its proportionality to the person's height.

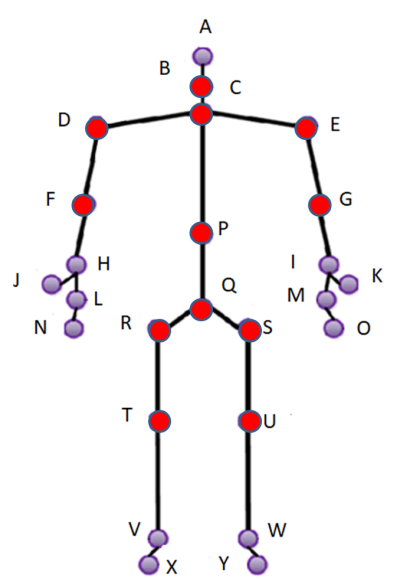

Fig. 2. The 3D joints defining the skeleton.

In addition to 3D joint distances, our joint-based features include geometrical angles extracted from every possible combination of three joints on the skeleton model. Geometrical angles defined by adjacent segments are directly estimated from joint positions in the 3D space. Once the body pose is modeled, posture recognition is performed using SVM classification. Figure 3 illustrates the main modules of the proposed system.

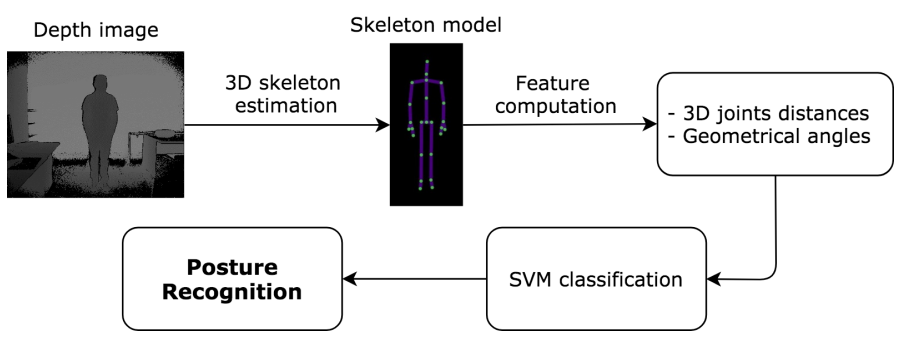

Fig. 3. Posture recognition using 3D joint-based features

\section{EXPERIMENTS}

\section{A. Data collection}

To evaluate the performance of the proposed methods, we created a new RGB-D dataset using the Microsoft Kinect v2 camera. The dataset includes the 3D joint coordinates in addition to RGB images and depth maps for each observation. To the best of our knowledge, this is the first posture recognition dataset created with the second version of the Kinect sensor (using Time of flight technology for depth sensing) ${ }^{1}$.

In our experimental setup, the camera is mounted on a tripod placed in a corner of our laboratory. Figure 4 illustrates five examples of body skeleton representing the five classes of postures: standing, bending, sitting, walking, and crouching. Each posture was performed by 13 participants of different ages, gender, and morphological characteristics. In order to

\footnotetext{
${ }^{1}$ The dataset will be published online upon acceptance
} 
increase the variability of our dataset and evaluate our system in handling scale and orientation change, each posture is captured at four different orientations and four different distances with respect to the camera. The distance varies from one to four meters while the orientation angle varies between 0 and 360 degrees. Our dataset includes a total number of 1040 observations that we used for training and testing the proposed system. For both methods, the dataset was split into two subsets, where $80 \%$ was used for training the systems, while the remaining $20 \%$ was exclusively used for testing.
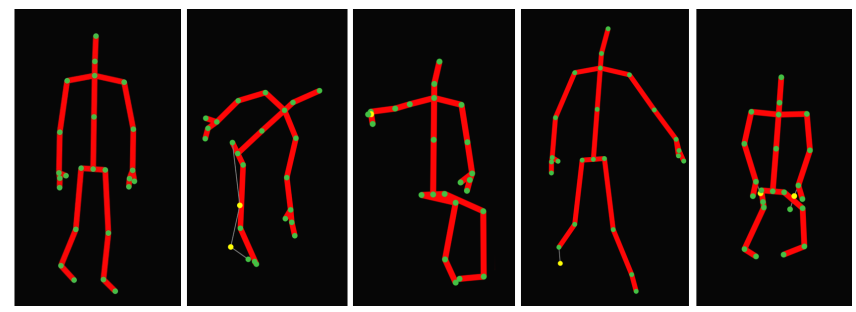

Fig. 4. Examples of body skeletons. From left to right : standing, bending, sitting, walking, and crouching.

All the experiments were implemented in Matlab and were executed on a machine with an Intel Core i7 $2.93 \mathrm{GHz}$ processor and $8 \mathrm{~GB}$ of RAM.

We designed our experiments in order to analyze the performance of our method according to several aspects.

\section{B. Experimental results}

1) Posture recognition using $2 D$ images and CNN: The first experiment aimed to identify the optimal image type, among RGB and depth images, for CNN training and classification. Table I presents the testing results for CNN models trained on segmented body silhouettes. The results show a slight superiority in accuracy for depth images.

TABLE I

VALIDATION AND TESTING RESULTS OF SEGMENTED RGB AND DEPTH IMAGES.

\begin{tabular}{|c|c|}
\hline \hline Image Type & Testing results \\
\hline \hline$R G B$ & $93.3 \%$ \\
\hline Depth & $95.7 \%$ \\
\hline
\end{tabular}

To further analyze the performance of the proposed methods, we used the confusion matrices. This evaluation tool provides a comparison of reference postures to the predicted postures. The rows of the matrices represent the true classes, while the columns represent the predicted classes. As shown in tables II and III, high recognition results were achieved for almost all the classes. We observed a 100\% accuracy for the bending, sitting and crouching classes. On the other hand, there was substantial confusion between the standing and walking classes with an accuracy rate of respectively $78.6 \%$ and $88.1 \%$ for RGB images, and $92.9 \%$ and $85.7 \%$ for depth images. This confusion can be explained by the visual similarity in the silhouette appearance between the standing and walking postures.
TABLE II

POSTURE RECOGNITION MATRIX: CNN CLASSIFICATION OF RGB IMAGES.

\begin{tabular}{|c|c|c|c|c|c|}
\hline \hline Postures & Standing & Bending & Sitting & Walking & crouching \\
\hline \hline Standing & $\mathbf{7 8 . 6 \%}$ & $0 \%$ & $0 \%$ & $21.4 \%$ & $0 \%$ \\
\hline Bending & $0 \%$ & $\mathbf{1 0 0 \%}$ & $0 \%$ & $0 \%$ & $0 \%$ \\
\hline Sitting & $0 \%$ & $0 \%$ & $\mathbf{1 0 0 \%}$ & $0 \%$ & $0 \%$ \\
\hline Walking & $11.9 \%$ & $0 \%$ & $0 \%$ & $\mathbf{8 8 . 1 \%}$ & $0 \%$ \\
\hline crouching & $0 \%$ & $0 \%$ & $0 \%$ & $0 \%$ & $\mathbf{1 0 0 \%}$ \\
\hline
\end{tabular}

TABLE III

POSTURE RECOGNITION MATRIX: CNN CLASSIFICATION OF DEPTH IMAGES.

\begin{tabular}{|c|c|c|c|c|c|}
\hline \hline Postures & Standing & Bending & Sitting & Walking & crouching \\
\hline \hline Standing & $\mathbf{9 2 . 9 \%}$ & $0 \%$ & $0 \%$ & $7.1 \%$ & $0 \%$ \\
\hline Bending & $0 \%$ & $\mathbf{1 0 0 \%}$ & $0 \%$ & $0 \%$ & $0 \%$ \\
\hline Sitting & $0 \%$ & $0 \%$ & $\mathbf{1 0 0 \%}$ & $0 \%$ & $0 \%$ \\
\hline Walking & $14.3 \%$ & $0 \%$ & $0 \%$ & $\mathbf{8 5 . 7 \%}$ & $0 \%$ \\
\hline crouching & $0 \%$ & $0 \%$ & $0 \%$ & $0 \%$ & $\mathbf{1 0 0 \%}$ \\
\hline
\end{tabular}

2) Posture recognition using $3 D$ joint-based features: Our first experiment set aimed to identify the optimal choices regarding the two main system components: 1) the feature set and, 2) the classification method. We thus investigated several choices entailing five classifiers (linear discriminant, quadratic discriminant, linear SVM, quadratic SVM, and cubic SVM), and three feature sets (geometrical angles, pairwise joint distances, and the combination of the 2 types of features). Table IV summarizes accuracy results for the tested classifiers applied to different feature sets. We can remark that the best recognition rates of linear discriminant and Fine KNN classifiers were obtained by using the 3D joints distances as features. However, SVM classifiers clearly outperformed both methods regardless the feature set. This investigation shows that the Quadratic SVM applied to the entire feature set achieves the best recognition rate $(93.1 \%)$. We therefore base our recognition system on 1) Quadratic SVM classification, and 2) a combination of the two types of features.

TABLE IV

PERFORMANCE RESULTS OF SEVERAL CLASSIFIERS USING DIFFERENT TYPES OF FEATURES.

\begin{tabular}{|c|c|c|c|}
\hline \hline Classifier & $\begin{array}{c}\text { Geometrical } \\
\text { angles }\end{array}$ & $\begin{array}{c}3 \text { D joints } \\
\text { distances }\end{array}$ & $\begin{array}{c}\text { Combined } \\
\text { features }\end{array}$ \\
\hline \hline Linear Discriminant & $83.3 \%$ & $87.4 \%$ & $83.2 \%$ \\
\hline Fine KNN & $84.5 \%$ & $87.7 \%$ & $86.2 \%$ \\
\hline Linear SVM & $89.2 \%$ & $89.8 \%$ & $91.2 \%$ \\
\hline Quadratic SVM & $92.3 \%$ & $92.3 \%$ & $\mathbf{9 3 . 1 \%}$ \\
\hline Cubic SVM & $91.7 \%$ & $92.0 \%$ & $92.5 \%$ \\
\hline
\end{tabular}

By analyzing the corresponding confusion matrix, it can be seen that the diagonal elements show high classification rates for all the classes. In table $\mathrm{V}$, the classification accuracy is higher than $90 \%$ for almost all the classes, except for the walking class $(89.9 \%)$. The highest classification accuracy was observed for the sitting class $(97.6 \%)$, since this posture is characterized by distinctive joint-based features. As with the first method, a confusion was also noted between the two 
classes walking and standing. However, the percentages of confusion are lower than those of the first method, which suggests that posture recognition using 3D joint-based features is more effective in distinguishing between the walking and standing postures.

TABLE V

POSTURE RECOGNITION MATRIX FOR QUADRATIC SVM CLASSIFICATION USING COMBINED FEATURES.

\begin{tabular}{|c|c|c|c|c|c|}
\hline \hline Postures & Standing & Bending & Sitting & Walking & crouching \\
\hline \hline Standing & $\mathbf{9 2 . 3 \%}$ & $0 \%$ & $0 \%$ & $7.7 \%$ & $0 \%$ \\
\hline Bending & $0.5 \%$ & $\mathbf{9 1 . 8 \%}$ & $1 \%$ & $4.8 \%$ & $1.9 \%$ \\
\hline Sitting & $0.5 \%$ & $0 \%$ & $\mathbf{9 7 . 6 \%}$ & $0 \%$ & $1.9 \%$ \\
\hline Walking & $8.6 \%$ & $0.5 \%$ & $1 \%$ & $\mathbf{8 9 . 9 \%}$ & $0 \%$ \\
\hline crouching & $0 \%$ & $1.0 \%$ & $5.3 \%$ & $0 \%$ & $\mathbf{9 3 . 7 \%}$ \\
\hline
\end{tabular}

\section{Discussion}

Due to their high accuracy and low computational complexity, both approaches can be applied for implementing practical applications, while meeting real-time requirements. However, several design choices depend on specific use cases and environmental constraints. For example, depth images lose their mapping quality at distances over three meters from the RGB-D camera. They are therefore inappropriate for open environments. We also observed a degradation in the silhouette segmentation quality on the two types of images in this situation. This can be explained by the fact that the segmentation method is highly dependent on depth maps.

From another point of view, posture recognition performed using depth maps and 3D skeleton model remain efficient regardless of lighting conditions, as the corresponding features only dependent on the infrared sensors of the RGB-D camera. This makes the system more robust and suitable for indoor environments where lighting condition is not stable. On the other hand, this type of environment represents a major limitation for RGB-based methods that are generally sensitive to light changes.

\section{CONCLUSION}

We presented novel solutions for automatic posture recognition using an RGB-D camera. We mainly designed two methods by exploiting different types of visual data provided by an RGB-D camera. The first method is based on CNN classification of 2D images, while the second uses 3D body modeling to compute and classify high level features. The proposed methods were validated on a challenging posture recognition dataset released in our laboratory. The experimental results showed comparable performances and high precision for both methods, with a slight superiority for the $\mathrm{CNN}$ based method when applied on depth images. Moreover both methods demonstrated a significant invariance to important perturbation factors, including scale and orientation changes.

This study offers several choices for implementing practical posture recognition systems, depending on the use cases and application constraints. As an example, methods using depth images are suitable for indoor environments with limited space. In such an environment, pose recognition can be efficiently achieved regardless the illumination condition.

\section{REFERENCES}

[1] B. Boulay, F. Bremond, and M. Thonnat, Human posture recognition in video sequence. IEEE International Workshop on VS-PETS, Visual Surveillance and Performance Evaluation of Tracking and Surveillance, 2003.

[2] T. Moeslund and E. Granum, 3D human pose estimation using 2D-data and an alternative phase space representation. Procedure Humans, 2000.

[3] A. Agarwal and B. Triggs, Recovering 3D human pose from monocular images. IEEE transactions on pattern analysis and machine intelligence, 28(1): pp. 44-58, 2006.

[4] I. Laptev, On space-time interest points. In International journal of computer vision (2005), Springer, pp. 107-123, 2005.

[5] I. Laptev, M. Marszalek, C. Schmid, and B. Rozenfeld, Learning realistic human actions from movies. In Computer Vision and Pattern Recognition, 2008. CVPR 2008. IEEE Conference on (2008), pp. 1-8, 2008.

[6] W. Bouachir, R. Gouiaa, B. Li, R. Noumeir Intelligent video surveillance for real-time detection of suicide attempts. In Pattern Recognition Letters, 2018.

[7] M. Zanfir, M. Leordeanu, and C. Sminchisescu, The moving pose: An efficient $3 \mathrm{~d}$ kinematics descriptor for low-latency action recognition and detection. In Proceedings of the IEEE International Conference on Computer Vision (2013), pp. 2752-2759, 2013.

[8] L. Xia, C. Chen, and J. Aggarwal, View invariant human action recognition using histograms of $3 \mathrm{~d}$ joints. In Computer Vision and Pattern Recognition Workshops (CVPRW), 2012 IEEE Computer Society Conference, pp. 20-27, 2012.

[9] G. Johansson, Visual perception of biological motion and a model for its analysis. In Perception \& psychophysics (1973), pp. 201-211, 1973.

[10] A. Tang, K. Lu, Y. Wang, J. Huang, and H. Li, A Real-Time Hand Posture Recognition System Using Deep Neural Networks. In ACM Trans Intell Syst Technol, vol. 6, no. 2, pp. 21:1-21:23, March 2015.

[11] P. Barros, S. Magg, C. Weber, and S. Wermter, A Multichannel Convolutional Neural Network for Hand Posture Recognition. In Artificial Neural Networks and Machine Learning ICANN 2014, Springer, Cham, pp. 403-410, 2014.

[12] A. Krizhevsky, I. Sutskever, and G. E. Hinton, Imagenet classification with deep convolutional neural networks. In Advances in neural information processing systems, pp. 1097-1105, 2012.

[13] J. Shotton, T. Sharp, A. Kipman, A. Fitzgibbon, M. Finocchio, A. Blake, M. Cook, and R. Moore, Real-time human pose recognition in parts from single depth images. In Communications of the ACM, 56(1): pp. 116-124, 2013.

[14] Transfer Learning Using GoogLeNet - MATLAB \& Simulink. [Online]. Available: https://www.mathworks.com/help/nnet/examples/transferlearning-using-googlenet.html. [Accessed: 29-Jun-2018].

[15] Transfer Learning Using AlexNet - MATLAB \& Simulink. [Online]. Available: https://www.mathworks.com/help/nnet/examples/transferlearning-using-alexnet.html. [Accessed: 29-Jun-2018]. 